\title{
La capacidad generativa de la familia. Un análisis sociológico desde el enfoque relacional y morfogenético*
}

\author{
Nuria Garro-Gil** \\ Universidad de Navarra, Navarra, España
}

\section{RESUMEN}

La sociedad occidental, especialmente, parece no valorar ni reconocer a la familia en toda su complejidad. La sociología, en tanto que disciplina que estudia la acción humana y sus formas de interacción social, está llamada a dar razones teóricas y prácticas válidas sobre el porqué de esta institución social. El objetivo de este artículo es tomar como punto de partida el enfoque relacional y morfogenético para proponer ciertas conceptualizaciones que permitan comprender qué puede significar actualmente ser y hacer familia. En este sentido, se propone la teoría relacional de Pierpaolo Donati como marco teórico desde el que repensar la actual crisis familiar y la pluralización de las formas familiares desde un punto de vista sociológico. Siguiendo un método analítico expositivo y desde un planteamiento teórico, se pretende: entender en primer lugar qué es y por qué existe la familia en un sentido sociológico, qué funciones desempeña y para qué es necesaria en la sociedad; analizar su estructura relacional y explicar la emergencia de nuevas formas familiares; valorar el potencial generativo que muestra la familia a nivel interno y externo; reconocer el papel clave que puede jugar en la creación y promoción de una cultura orientada al cuidado de las personas y el entorno.

* Este artículo forma parte de una investigación teórica más amplia acerca de la sociología relacional de Pierpaolo Donati, desarrollada en el período 2010-2014, y que dio como resultado la tesis doctoral inédita titulada "La relación de cuidado en la familia", defendida en la Facultad de Educación y Psicología de la Universidad de Navarra (Espańa), en junio de 2014, calificada con sobresaliente cum laude. Dicha investigación fue financiada por la Asociación de Amigos de la Universidad de Navarra (España) y cofinanciada por la Fundación Caser para la Dependencia de Madrid (Espańa) dentro del proyecto de investigación "La relación de cuidado en la familia" (2014-2016, investigador principal, Dr. Alfredo Rodríguez Sedano).

** Doctora internacional en Pedagogía, Universidad de Navarra, España. En la actualidad es ayudante doctora, Facultad de Educación y Psicología, en la misma universidad. Correo electrónico: nggil@ unav.es. 


\section{Palabras clave}

Familia, sociología relacional, enfoque morfogenético, transferencia social, cultura de cuidado

The generative capacity of the family. A sociological analysis from the relational and morphogenetic perspective

\section{ABSTRACT}

Western society, especially, seems not to appreciate or recognize family in all its complexity. Sociology, as a discipline that studies human action and forms of social interaction, is called upon to give valid theoretical and practical reasons about the motive of this social institution. The aim of this article is to take, as a starting point, the relational and morphogenetic approach to propose certain conceptualizations for understanding what being and making a family can actually mean. In this sense, the relational theory of Pierpaolo Donati is proposed as a theoretical framework from which to rethink the current family crisis and the pluralization of family forms from a sociological point of view. Following an analytical method and from a theoretical approach, we aim to understand what the family is and why it exists, in a sociological sense, what functions does it perform and why is it necessary in society; analyze its relational structure and explain the emergence of new family forms; show and assess its internal and external generative potential; recognize the key role it can play in the creation and promotion of a culture that is oriented toward caring for people and the environment.

\section{Keywords}

Family, relational sociology, morphogenetic approach, social transfer, caring culture

\section{Introducción}

Hasta mediados del siglo XIX se acepta de modo general entre los teóricos de la familia que en los orígenes de la humanidad ya existe como forma fundamental el matrimonio monogámico. Bachofen (1992) marca un antes y un después en la ciencia y en la filosofía con la publicación de su obra en 1861 sobre el matriarcado o el 'derecho materno', considerado el primer intento de hacer una historia científica de la familia como institución social. Por entonces, la antropología social, la arqueología, la historia y la etnología, principalmente, manifiestan la enorme diversidad en 
cuanto a formas de organización familiar y parentesco a lo largo de la historia. Esto plantea a la ciencia un doble reto, sobre todo a nivel metodológico: 1) establecer una cronología de las formas y cualidades familiares, y 2) definir los procesos evolutivos en las formas de organización familiar y parentesco (Martín López, 1993).

A partir del siglo XX comienzan a surgir nuevas investigaciones, principalmente etnográficas, que rechazan el sentido evolucionista y la generalización indebida de hallazgos y adoptan un mayor rigor metodológico. Pretenden aportar una explicación no lineal de los procesos de transformación de las instituciones sociales y evitar además el relativismo y el particularismo histórico (Boas, 1964), lo cual viene a cuestionar esas primeras investigaciones con origen en Bachofen. Posteriormente, la antropología abandonará la discusión sobre la evolución de las formas familiares y de parentesco, y situará el estudio de formas aisladas y singulares en sociedades concretas (Martín López, 1993). Sociólogos y antropólogos comienzan entonces a plantear la objetividad de su estudio manifestando una preocupación común: la excesiva proximidad del observador a su objeto de estudio y la dificultad concreta de las ciencias humanas para alcanzar esa objetividad (Martín López, 1993). Con ello se sientan las bases del estudio empírico y funcionalista del sistema familia.

La sociología de la familia comienza, pues, a asumir una visión funcionalista, según la cual la familia no es sino la elección individual que resulta del sentir subjetivo, y es por tanto una cuestión que atańe únicamente al ámbito privado (Belardinelli, 2013). Lo que se cuestiona en ese momento es la identidad de una institución social que parece evolucionar indefinidamente hacia formas convivenciales cada vez más diversas. Así comienza a difundirse durante la modernidad la idea de que la familia 'tradicional' está desapareciendo y se pone en duda la vigencia de un modelo que se considera obsoleto y anticuado, con pocas posibilidades de cambio y por lo tanto culturalmente descalificado (Donati, 2014). A todo ello contribuyen también los medios de comunicación y diversas teorías sociales que se hacen eco de la crisis familiar y su inminente desaparición (Belardinelli, 2013).

La sociología relacional de Pierpaolo Donati $(1983,1986,1987,1989)$ emerge en el escenario sociológico contemporáneo como una teoría novedosa y esperanzadora al resituar el foco de atención en la relacionalidad de la familia y los bienes particulares y únicos que esta genera y transfiere a la sociedad. El sociólogo italiano comienza señalando la falta de base empírica en las afirmaciones que anticipan la muerte de la familia y en la generalización estadísticamente significativa de otras formas familiares dominantes que tengan continuidad en el tiempo. El objetivo, dice, es analizar si efectivamente se trata de una sensación difundida pero sin fundamento científico o, por el contrario, la familia está llamada a desaparecer en los años venideros (Donati, 2014). 
La investigación sociológica muestra, de hecho, que la formación de familia sigue siendo objetivo vital de las nuevas generaciones, como así muestran estudios realizados en este nuevo siglo en torno a los valores de adolescentes y jóvenes (García Garrido et al., 2000). Si bien no deja de ser cierto que en la actualidad parecen redefinirse los términos del proyecto de vida en común y este adopte un carácter más negociador (Ayuso Sánchez, 2010). La tesis que plantea Donati es que, efectivamente, la familia se enfrenta hoy a grandes procesos de diferenciación. Pero estos, lejos de aventurar su desaparición, muestran el proceso de morfogénesis que obliga a: por un lado, repensar la relación-familia respecto del modelo tradicional; $y$, por otro lado, distinguir la familia normo-constituida respecto de las formas plurales que actualmente emergen manifestando estructuras y organizaciones de todo tipo.

No cabe duda de que la sociedad actual, especialmente en Occidente, manifiesta un cierto escepticismo respecto de la funcionalidad y utilidad de la familia tradicional (Del Campo, 2006). Y es por eso que la sociología está llamada a dar razones teóricas y prácticas válidas sobre el porqué de esta institución social, sobre el hecho de tener una forma determinada y no otra, y sobre su posible continuidad. Por ello, y para no quedarse en consideraciones de orden filosófico o moral, como es habitual entre las teorías tradicionalistas (Belardinelli, 2013), Donati propone dar respuesta a la pregunta de qué es la familia basándose en hechos, en realidades sociológicas. El reto que se le plantea a la teoría relacional es mostrar cuáles son esas cualidades y poderes propios de las relaciones familiares que hacen que sea esa forma social y no otra la que a lo largo de la historia haya sobrevivido como sociedad natural dominante en gran parte de las culturas (Donati, 2014).

El presente artículo pretende mostrar la familia como relación-raíz de la sociedad desde el punto de vista sociológico y tomando como fundamento teórico el enfoque relacional de Donati y la utilización que él mismo hace del enfoque morfogenético de Archer (2007). El objetivo es analizar cómo es esa estructura relacional que llamamos familia y que conforma una realidad sui géneris cuya naturaleza reside en su genoma social. Asimismo, entender su capacidad generativa para crear bienes o males relacionales que ninguna otra institución o sistema social es capaz de generar y que son transferibles a la esfera pública a través de la socialización de las nuevas generaciones, lo cual tiene un impacto en las relaciones y los intercambios sociales que pueden ser la base de una sociedad más humanizada y transformadora, orientada al cuidado.

Tratándose de un análisis sociológico, la teoría relacional de Donati destaca asimismo por su carácter interdisciplinar, puesto que introduce conceptos y cuestiones que limitan con la antropología, la filosofía, la ética, la política social y 
otras disciplinas afines. Esto resulta de especial interés al tratar un tema como es la familia, siempre transversal y presente directa o indirectamente en casi todas las cuestiones que versan sobre el hombre y la sociedad. Es de esperar, por tanto, que la propuesta teórica que aquí se presenta pueda realizar un aporte de interés al campo de los estudios sobre familia.

\section{La pluralización de las formas familiares}

La sociología contemporánea se enfrenta al reto de definir qué es familia en medio del debate social que cuestiona su identidad y sus funciones (Belardinelli, 2013). De hecho, la sociedad posmoderna llega a asumir e integrar en su imaginario colectivo una idea de familia que contribuye de forma progresiva a su desaparición tal y como se ha concebido hasta ahora. De acuerdo con los ideales de libertad, pluralismo y tolerancia, la familia debe mostrarse también como la elección subjetiva que cada quien realiza de entre "una oferta excesiva de posibilidades de elección" (Beck, 1996, p. 76). Y los medios de comunicación contribuyen a difundir y generalizar la creencia de que las relaciones familiares comienzan a perder densidad y estructura. Son, en palabras de Bauman (2001), relaciones virtuales en las que tan fácil es entrar como salir, a través de las cuales se crean vínculos precarios y transitorios.

Frente a las corrientes actuales, la sociología relacional de Donati intenta dar un paso más allá y plantea si la familia es una institución obsoleta que pertenece al pasado. $\mathrm{O}$, por el contrario, posee una estructura sui géneris respecto de la cual se puede medir el carácter más o menos humano y humanizador de las relaciones sociales (Donati, 2014). El mismo lenguaje indica la posible existencia de una naturaleza concreta desde la cual se proyecta la pluralidad de formas convivenciales aparentemente equivalentes: se habla de 'la' familia y no de 'las' familias (Donati, 2014); en todo caso se dice 'modelos de familia'. La pregunta es si el concepto 'familia' representa la existencia de un principio que sea origen del resto de formas emergentes.

Como punto de partida, Donati (2014) identifica dos modelos principales desde los que la sociología intenta explicar los cambios sociales que afectan a la familia: el modelo tradicionalista y el modelo evolucionista. Las teorías tradicionalistas (Berger y Berger, 1983) mantienen que la pluralización de las formas es solo una tendencia negativa que resulta autodestructiva y regresiva, y que en todo caso manifiesta la degradación de una sociedad incapaz de solucionar los problemas ni aun generando nuevas formas de agrupación. Estas teorías pretenden reducir y hasta negar la pluralización y recuperar el modelo tradicional de familia, incluso 
a costa de poder analizar y prever dinámicas sociales que permitan anticipar los cambios que no dejan de ser evidentes. Por el contrario, las teorías evolucionistas - de corte funcionalista- muestran cómo la familia está llamada a extinguirse como resultado de un proceso evolutivo sociocultural y subjetivo más o menos determinista que desemboca en el pluralismo de formas más aptas y funcionales, respecto de las cuales la capacidad de adaptación se impone como valor.

D’Agostino (1991) identifica también dos modelos principales desde los cuales se ha estudiado y descrito la familia, que él denomina modelo tradicional y modelo moderno. El modelo tradicional, de corte más holista, ha dado prioridad a la estructura familiar por encima de los individuos, constituyéndose así como un sistema cerrado y privado donde gobierna el dominio masculino. El modelo moderno de corte más individualista refuerza la primacía de los individuos que componen la familia, creando un sistema más abierto al exterior a través de la individualidad de sus miembros. Concluye que ambos modelos son criticables no tanto por el hecho de si son más o menos modernos, sino porque el primero elimina la dimensión personal y el segundo peca de utilitarismo y privatización de los intereses individuales -principalmente de los cónyuges. Ambos modelos ignoran el sentido de comunidad que da fundamento y constituye la familia (Naval, 2003) y les lleva a absolutizar una sola de las muchas verdades del vínculo familiar en detrimento de las restantes.

También Belardinelli (2013) señala la limitación que presentan los modelos teóricos dicotómicos que él clasifica en 'católicos' y 'laicos', y cuyas respuestas unilaterales y reduccionistas no dan solución satisfactoria al problema de definir la familia y entender los cambios que experimenta.

En todos los casos, el error común de fondo parece ser, según Donati (2014), la utilización del término 'tradicional' para referirse a la institución familiar, ya sea en señal de rechazo o de reconocimiento, atribuyéndole un carácter histórico y universal a un modelo familiar que en realidad está históricamente situado en una época y sociedad concretas. Por lo tanto, los cambios en el modelo 'tradicional' de familia no deben tomarse únicamente en un sentido negativo, como eliminación de la familia, sino como la superación de las estructuras propias de una época pasada, posiblemente desfasadas, y que, en todo caso, han creado relaciones inter e intrageneracionales no siempre deseables: autoridad exclusivamente paterna, obediencia de la mujer frente al hombre, rol femenino limitado al ámbito doméstico, figura del padre autoritario y lejano respecto de los hijos, relaciones filiales distantes y jerárquicas, fuerte cohesión de una familia nuclear más cerrada al exterior y convivencia simultánea de varias generaciones (Donati, 2013). 
La misma cuestión señalaba Héritier (1984) cuando decía que la muerte de la familia hacía referencia al modelo burgués. Implícitamente afirmaba que existe una forma 'ideal-eterna' de familia presente en todas las concreciones históricas y además capaz de reencarnarse en formas futuras (D’Agostino, 1991). Esta misma idea es la que plantea Donati (2009) cuando habla de la dualidad ontológicofenomenológica que apunta a la familia como relación-raíz - lo que la familia es por naturaleza- y relación-contingencia -cómo se manifiesta histórica y temporalmente en una sociedad concreta adoptando formas diversas.

Donati termina por rechazar las teorías tradicionalistas y funcionalistas, y concluye que en realidad el modelo tradicional de familia es utilizado como "cómodo estereotipo polémico" (Donati, 2014, p. 29), aceptado por unos y rechazado por otros. Pero, en medio del debate social, ni unos ni otros parecen darse cuenta de que su error común es tomar como referente un modelo de familia que no es sino una concreción histórica, temporal y espacialmente situada, posiblemente poco representativa ya -al menos en algunos aspectos- de los retos y desafíos actuales. Pero que, sin embargo, remite a una naturaleza latente y continua que está siempre presente en cada una de esas formas emergentes, como principio generativo. Y esa naturaleza latente de la familia es el objeto de estudio de la sociología relacional para entender el pasado, presente y futuro de la familia como institución social.

Analizando el modo como la sociología ha intentado definir a la familia, Donati percibe que la perspectiva adoptada habitualmente desde un enfoque histórico ha llevado no tanto a examinar 'qué' es la familia, sino a qué se le ha dado el nombre de familia y cómo ha sido concebida en los distintos períodos, culturas y ámbitos sociales (Caporrella, 2008). Así lo muestran los estudios iniciales que analizaban el cambio social en la familia europea y planteaban las distintas alternativas a la familia nuclear, centrándose en los aspectos objetivos de sus cambios estructurales y organizativos: tamaño, número de miembros, nupcialidad, natalidad, mortalidad, rupturas, formas de cohabitación, etc. (Díez Nicolás, 1983).

La familia se enfrenta en la actualidad, más que nunca, a un creciente proceso de desinstitucionalización y a la emergencia de muchas formas y estructuras sociales aparentemente equivalentes que parecen poder asumir y desempeñar igual o más eficazmente las funciones familiares. Quizás la clave resida en entender el tipo de relaciones que conforman la familia y cuya naturaleza parece mantenerse estable más allá de cualquier cambio sociocultural. Para ello parece necesario revisar y repensar desde un punto de vista teórico qué entendemos hoy por familia y qué representa en la actualidad esa institución social que, queramos o no, ha permanecido invariable en el tiempo en lo que a su principio generativo se refiere. 


\section{La familia como problema sociológico: entender la sociabilidad más allá de la socialización}

Las teorías que plantean la pluralización de las formas familiares olvidan que la institución familiar manifiesta una realidad sui géneris respecto de la que se mide el carácter más o menos humanizador de la sociedad (Donati, 2013). Es decir, si el objetivo en el nuevo siglo es hacer más humana esa sociedad que muestra dificultades para distinguir lo humano de lo no-humano, esta no puede existir si no existe la familia. Los motivos que justifican tal afirmación es que la familia es, en definitiva, generadora de capital humano. De acuerdo con su propia estructura interna que opera por y a través de relaciones, la familia se convierte en fuente de sociabilidad en tanto que genera bienes relacionales - relaciones que contribuyen al crecimiento y felicidad de las personas- que transfiere a la sociedad y contribuye a crear relaciones sociales más humanas; en definitiva, más familiares. Por eso, la reflexión en torno a la relación-familia plantea una nueva forma de entender la sociabilidad más allá de la mera socialización -adaptación de los individuos a las estructuras- como expansión de la solidaridad (Bernal, Rodríguez, Altarejos y Naval, 2005). Así lo describe Belardinelli (2013) cuando dice que la familia representa ese primer banco de prueba de la antropología relacional: el encuentro del hombre con la alteridad -con los otros y con el mundo que le rodea- le hace consciente de que no vive solo.

Donati (2013) señala en este sentido que gran parte de las patologías actuales vienen dadas precisamente por problemas que tienen que ver con no saber ser y hacer familia, y con generar males relacionales que posteriormente condicionarán la socialización de las personas y las relaciones sociales. Por eso insiste en entender la familia como vínculo y también como recurso en una sociedad cada vez más instrumentalizada, en la que las relaciones de interés, de utilidad, relaciones tóxicas, destructivas, parecen generalizarse.

Tal y como apunta D’Agostino (1991), el primer paso para la reinstitucionalización de la familia es entender que ella no es el resultado de la intencionalidad subjetiva o de los determinismos estructurales. La familia no es consecuencia de un simple querer o apetecer, que es de suyo inestable puesto que depende de sentimientos. Tampoco deriva de una simple estructura; de ahí que la expresión 'familias estructuradas y desestructuradas' resulte bastante reduccionista si nos quedamos únicamente con la idea de que la estructura por sí sola puede hacer familia (Cabezas, 2008). La familia remite en realidad a una estructura antropológica. Esta se manifiesta a nivel fenomenológico bajo el condicionamiento de las estructuras y los cambios socioculturales, pero en relación siempre a un mismo principio: 
La novedad [...] es que la familia constituye una comunidad de amor y de solidaridad: una comunidad que no encuentra su fundamento último ni en la ley que le otorga la reglamentación, ni en la utilidad que pueden extraer de ella sus componentes, sino en la capacidad (en sí misteriosa, pero indudablemente típica del hombre) de amar familiarmente y de fundar sobre este amor una comunidad de vida. (D’Agostino, 1991, p. 18)

[M]ás allá de las inevitables interacciones con el contexto socio-cultural, la estructura de la familia no es sólo socio-cultural, sino antropológica: es decir, que dicha estructura no atañe sólo al orden empírico de la coexistencia, sino que radica en una exigencia estructural del ser mismo del hombre. [...] [Las necesidades que manifiesta el hombre] surgen de las más profundas exigencias del ser del hombre, que pide amor y puede dar amor, que pide solidaridad y puede dar solidaridad. $\mathrm{Al}$ advertir a la familia como comunidad de amor y de solidaridad no sólo se conquista el reconocimiento de las expectativas irrenunciables de dicha familia, sino que también se acepta una imagen del hombre muy determinada: la única en la que puede apoyarse la esperanza de una sociedad y de un futuro no inhumanos. El hombre que se abra al amor familiar y reconozca los valores personales y de don pertenecientes a dicho amor, es también un ser humano capaz de construir y defender una escala de valores coronada por la dimensión comunitaria solidaria y capaz, por ello, de controlar las pulsiones egoístas, violentas y, a fin de cuentas, destructivas que el hombre sin duda lleva consigo y que ninguna forma de política social podría nunca sofocar. (D’Agostino, 1991, pp. 21-22)

De acuerdo con esta reinterpretación de la pluralización de las formas, la teoría relacional propone mostrar que la familia se enfrenta en la actualidad a procesos de diferenciación que la cuestionan en su misma raíz o estructura, poniendo en duda su utilidad y relevancia social (Donati y Colozzi, 1997). El problema reside en que todavía no se ha sabido definir desde un punto de vista sociológico qué es la familia. No se ha identificado su estructura interna ni su relacionalidad, que es lo que en definitiva hace de ella un sistema social sin equivalentes funcionales. Tampoco se ha sabido interpretar que los cambios sociales que la condicionan y modifican no hacen sino diferenciarla y reforzarla como institución social, mediadora y por tanto educadora. Por ello es necesario adoptar un enfoque teórico que sea capaz de observar la familia como relación, que genera y alimenta un entra- 
mado único de relaciones, e interpretar los cambios y procesos de diferenciación que experimenta desde un punto de vista relacional.

\section{La familia en la sociología relacional}

El problema actual al que se enfrenta la familia es la pérdida de identidad: no se sabe qué o quién es, ni en qué se diferencia realmente del resto de formas emergentes. La teoría relacional aporta una nueva luz al señalar que su identidad reside precisamente en una relación social que, aun siendo inmaterial e invisible al ojo humano, posee entidad y manifiesta por lo tanto una realidad propia, sui géneris: su realidad consiste en un tejido de elementos subjetivos -intencionalidad- y objetivos -estructura vinculante- que trascienden las cosas ya dadas -reciprocidad generativa- (Donati, 2013).

El acercamiento relacional [...] afirma que la familia pertenece a un orden de realidad social sui generis que otras disciplinas (desde la filosofía a la economía) todavía no han comprendido. [...] la familia es más bien una configuración relacional que va más allá de la simple suma de individuos y no llega jamás a ser un cuerpo orgánico, lo cual quiere decir que no agota nunca sus posibilidades. (Donati, 2013, p. 31)

Esta relación conecta así dos aspectos centrales: por un lado, la relación conyugal (hombre-mujer), que es el primer núcleo relacional y generativo de la familia, y por otro, la relación filial, en la que los hijos son considerados el bien relacional primario, fruto de dicha unión. Todo ello es reflejo de la trascendencia de la individualidad que se abre a un proyecto común y es garantía de continuidad de la especie humana (Gil y Arias, 2013).

La teoría relacional señala también que la relación-familia es generadora de lo social y, más allá de sus relaciones internas, da vida también a relaciones sociales verdaderamente 'familiares' que son germen de una sociedad civil solidaria y comprometida actualmente en auge (Donati, 2013). En este sentido, la familia da muestra de dos cualidades propias de su naturaleza relacional (Donati, 2014):

- Es originaria: nace de las motivaciones e impulsos propios, no solo como resultado de presiones o factores externos a la relación. Es decir, la sociedad no hace familia, sino que la familia es, y es ella la que hace sociedad.

- Es original: se despliega desde su propia lógica o código simbólico de in- 
tercambio que es el de la plena reciprocidad entre sexos y entre generaciones; el intercambio como don, basado en el amor, que trasciende toda utilidad o interés individual.

Ante la pregunta que plantea la sociología de por qué y para qué existe la familia, la teoría relacional responde: para satisfacer las necesidades humanas relacionales (Donati y Solci, 2011). La persona, desde que nace, necesita de las relaciones familiares que le sirven de punto de apoyo e incluso de campo de entrenamiento para posteriormente entrar a formar parte de una sociedad global, compleja y desafiante. Pero no solo los individuos necesitan de la familia, también la sociedad y el resto de los sistemas precisan sus funciones de medicación.

Las funciones en el seno de la familia, entre cónyuges y entre generaciones, son un reflejo de modelos de autoridad, así como de cooperación horizontal y jerárquica, y reflejan también relaciones entre lo individual y lo colectivo que obviamente influyen en las funciones y relaciones que se dan en la sociedad en general, a la vez que son influidas por ellas. (Putnam, 2003, p. 319)

En definitiva, la familia es ese ámbito natural en el que el individuo encuentra respuesta a sus necesidades e intencionalidades, motivaciones subjetivas autónomas y deseos de realización y proyección; y es también el sistema ante el cual la sociedad puede plantear sus exigencias funcionales y suprafuncionales (Donati, 2014).

Una vez que se ha entendido que la familia manifiesta una naturaleza singular que se despliega en la contingencia de lo social, su desinstitucionalización puede ser interpretada bajo otro prisma: está cambiando su relacionalidad constitutiva y existe una dificultad manifiesta para identificar y diferenciar la relación-familia del resto de las relaciones. Como punto de partida, Donati (2013) identifica tres formas diferentes en las que se viene utilizando indistintamente el término familia:

- Familia como metáfora: se basa en la similitud que mantienen las diferentes formas con la familia, con la que comparten alguna cualidad, aunque manifiestan naturalezas distintas. Son, por ejemplo, las comunidades religiosas.

- Familia como analogía: se basa en la existencia de semejanzas concretas respecto de la familia; sin embargo, estas formas no son capaces de generar el intercambio de plena reciprocidad que la caracteriza. Es el caso de las diversas formas convivenciales.

- Familia como identidad: manifiesta una identidad específica que se distingue por su cualidad única de plena reciprocidad entre los sexos y las generaciones. 
La siguiente pregunta sería: ¿qué es aquello que distingue a la familia en su sentido identitario como forma originaria y original del resto de las formas metafóricas o análogas que emergen en relación a ella? La respuesta es que lo distintivo de la familia es la capacidad que manifiesta para hacer estables, previsibles y socialmente tutelables los intercambios entre géneros y generaciones (Donati, 2014). Es decir, la familia genera expectativas; hace posible que se espere de ella, y que se esperen relaciones y funciones concretas, de la forma como solo ella las genera, las transfiere socialmente y las asume.

Igualmente conviene analizar el proceso evolutivo que ha experimentado la institución familiar en el transcurso de su desarrollo, puesto que, como manifiesta Donati (2013), este no es un fenómeno propio únicamente de la sociedad actual, sino que ha estado presente a lo largo de la historia como proceso que ha acompañado siempre al cambio social. El sociólogo identifica cuatro semánticas distintas:

- Tradicional: propia de culturas premodernas y estables que fundan la familia sobre la base étnica o religiosa. Son formas plurales, diferentes entre sí, que presentan identidades fijas, estables y sólidas, con poca o nula variación.

- Moderna: la identidad familiar se diversifica desde la idea de contingencia social. Queda sujeta a la ciencia y el derecho positivo, de manera que se hace históricamente variable. Son las instancias médico-tecnológicas o jurídicas las que dictaminan qué es familia.

- Posmoderna: hace contingente todo aspecto de la familia y su regulación es únicamente responsabilidad de los propios individuos que la conforman, siguiendo la norma de mantenerse éticamente neutro, otorgando a todo resultado igual valor. La pluralidad es sinónimo de desorientación y disgregación, y la indiferenciación adquiere un doble significado: como indeterminación de las formas e indiferencia de quien las observa.

- Dopomoderna: es la cuarta semántica que la teoría relacional propone para la reinterpretación de la pluralidad de la familia contemporánea. "[L]a pluralidad dopo moderna será el producto de una diferenciación que valorará las relaciones familiares como cualidades distintivas de un sentido de la vida (y de los recursos, ante todo humanos) que no cabe encontrar en otras relaciones" (Donati, 2014, p. 37).

De acuerdo con la semántica dopomoderna, la excesiva nuclearización que habitualmente se le reconoce a la familia contemporánea (Fernández Muñoz, 2000) y la desaparición paulatina de la familia extensa (Crespo y Moretón, 2010) cobran aquí una nueva perspectiva. Se constata la desestabilización del núcleo familiar, pero también se consolidan y crean complejas tramas de relaciones en las que la 
familia interactúa con tantas otras personas y núcleos familiares, así como con sistemas sociales diversos. Se trata, por tanto, de un proceso de aparente privatización creciente pero al mismo tiempo de mayor apertura al exterior, creando así nuevas redes relacionales (Donati, 2014). Todo ello lleva a la sociología relacional a rechazar hablar de crisis familiar y apostar por un enfoque morfogenético para analizar los cambios que está experimentando. "[E]stos fenómenos existen e indican que ciertos grupos sociales de familias podrían organizarse en líneas de agregación en red, de solidaridad a distancia, de intercambios orientados a nuevas sinergias entre los sexos y las generaciones" (Donati, 2013, p. 16). Lo que urge entonces examinar es el sentido que adopta dicho proceso de pluralización (Donati, 2013):

- Como patología social: en cuyo proceso se da un pluralismo moralmente indistinto, que no hace diferencias y valora de forma neutral todas las transformaciones. - Como proceso que forma parte de la fisiología de la familia y que es moralmente cualificado: de tal forma que las transformaciones familiares son algo positivo, como muestra de la rica pluralidad que manifiesta la familia.

En definitiva, para hacer una interpretación sociológica compleja y no reduccionista de los procesos de pluralización, la teoría relacional propone tener en cuenta dos grandes ejes descriptivo-interpretativos articulados entre sí relacionalmente (Donati, 2013):

- Una semántica distintiva de la pluralidad que, como ya se ha visto, corresponde a la diferenciación de las relaciones familiares a través de una racionalidad relacional centrada en la calidad humana de las relaciones, y no funcionalista o instrumental más centrada en su interés o utilidad.

- Una teoría de la morfogénesis familiar: la cual propone repensar y analizar la familia y sus cambios desde el punto de vista de la generación de formas que pueden indicar un cambio más profundo en su principio generativo o naturaleza relacional.

El enfoque morfogenético que adopta la sociología relacional permite interpretar la pluralidad familiar desde un punto de vista relacional más allá de la desinstitucionalización que anuncia la modernidad (Beck y Beck-Gernsheim, 2002), entendiendo por morfogénesis (Archer, 2007) aquel proceso que posee una dinámica concreta, descriptible y observable en el tiempo, manifestando una interdependencia o condicionamiento recíproco entre factores identificables y no causales, los cuales se mueven según la lógica de vínculos y recursos, y que debe responder, en un cierto sentido, a exigencias de sostenibilidad. De esta manera, 
los cambios que acontecen dentro y fuera de la familia pueden generar también estructuras y sistemas relacionales que apuntan a modificaciones en su principio generativo y que por tanto le confieren un nuevo sentido institucional y le atribuyen un estatus social distinto (Donati, 2014, 2008a):

- La pluralidad de la familia puede aumentar o disminuir en intensidad y en cantidad a lo largo de la historia. Pero su aumento no es indicativo de que la familia pierda relevancia social. Más bien refleja la necesidad de poner en marcha nuevos procesos de diferenciación que la distingan de todas aquellas formas que en realidad responden a una naturaleza distinta. Es decir, conviene identificar y distinguir el tipo de relaciones que solo la familia es capaz de generar y transferir después a la sociedad.

- La pluralización es manifestación de una distinta articulación relacional de los ejes sobre los que se forma la familia: la relación conyugal y la relación filial. Esas formas emergentes surgen a partir de procesos morfogenéticos -generación de nuevas formas de relación- que articulan las relaciones y conjugan los elementos -intencionales y estructurales- de múltiples maneras.

- La pluralidad casi ilimitada de las formas es también resultado de variaciones en el genoma social de la familia según se articulan y relacionan de una manera u otra las cuatro dimensiones que lo constituyen, como se verá más adelante.

En definitiva, el reto que se le plantea a la sociedad en el siglo XXI es "profundizar en las distinciones entre las relaciones propiamente familiares y las no-familiares" (Donati, 2013, p. 10).

\section{La estructura relacional triádica}

Esta nueva forma de interpretar y comprender la pluralización desde el enfoque relacional y morfogenético, indica la existencia de una estructura latente en la naturaleza de la familia, entendiendo por latente aquello que permanece oculto, escondido, pero que es raíz y origen del ser. "[T] oda relación es contingente (en el doble sentido de poder no-ser, por tanto de poder ser de otra manera y de depender de otro de sí)" (Donati, 2010, p. 14). También la familia es raíz, estructura latente de la sociedad, que podría no-ser, pero que de hecho es. En su caso se trata de una estructura triádica que se forma sobre la base de su circuito de reciprocidad plena: donación-aceptación-don. 
Que la sociología tenga en cuenta la relación trinitaria permite observar el sentido generativo, mediador y reversible de la relación, pero no supone asumir ningún valor o ideología a priori, manteniendo siempre la neutralidad metodológica, ni requiere tampoco creer en la trinidad. (Donati, 2010, pp. 21-22)

La donación-aceptación-don es el intercambio recíproco que se da entre sujetos complementarios, quienes desde su propia condición sexuada buscan el encuentro y el intercambio con el otro. La unión a la que dan lugar conforma una díada con capacidad natural generativa de la que resultan los hijos, abierta por tanto a la trascendencia de la pareja (Donati, 2003). La díada se convierte así en una tríada que conecta géneros y generaciones, representada por las relaciones esponsales y filiales (Terenzi, 2006). Dicha estructura se identifica con la "plenitud relacional" (Donati, 2013, p. 19): es la característica propia de las relaciones familiares que son capaces de conectar entre sí a los sujetos con la 'totalidad' de su ser (Marías, 1989), más allá del rol y función que desempeñan. Ahí reside el sentido propio y pleno de la realidad que llamamos familia desde un punto de vista sociológico. Las personas interesan y son acogidas y queridas por quienes son, y no por lo que son. Siempre y cuando, claro está, la familia en cada caso concreto genere aquellas relaciones que está llamada a generar.

Donati (2013) distingue en torno a esta cuestión entre lo que él denomina:

- Parejas agregativas: centradas en la pareja y no abiertas a la generatividad. Podría ser la 'relación pura' de la que habla Giddens (1992) como forma contractual opuesta al amor romántico (De Rougemont, 2006), en la que predominan la desinstitucionalización y la individualización, y donde las relaciones siguen la regla de la paridad de cuentas en el dar y el tener. Podría ser también la 'pareja de razón' de Roussel (1980), basada en el consenso y la asociación en la cual prevalece la negociación de intereses individuales (Belardinelli, 1996). Son relaciones generadoras de estilos de vida en las que el principio subjetivo introduce la posibilidad siempre presente de que la pareja pueda disolverse a través de "contratos revisables" (Belardinelli, 1996, p. 57).

- Parejas generativas (abiertas a ella). Esto no queda en una mera cuestión procreadora, sino que pone de manifiesto intencionalidades, vínculos, estilos de vida y relaciones diversos. Y con implicaciones no solo en la propia relación familiar y en la socialización de sus miembros, sino también en las propias dinámicas sociales, precisamente como consecuencia de la transferencia social de los bienes o males relacionales que la familia es capaz de generar. 
La forma familiar de la relación coincide con una elección interpersonal de amor conyugal que se abre a un proyecto arriesgado de vida común, que busca su plenitud en el regenerar continuamente un vínculo que significa vivir para el otro. El don se hace en este circuito de reciprocidad, la relación sexual es querida como una que tiene un carácter especial en vista de una generación (este hijo y no otro), que es expresión de esa relación y no de otra. En este compartir-coexistir, hecho de referenciassignificados y de conexiones-vínculos-nexos, emerge esa relación de pertenencia que llamamos "familia". (Donati, 2013, p. 18)

La relación-familia manifiesta una triple estructura que hace converger 1) una intencionalidad -re-fero- libre y subjetiva de dos personas que se dirigen el uno al otro y eligen mutuamente para crear una unión generativa que da lugar a la díada; 2 ) una vinculación estructural -re-ligo-sociocultural y normativa que regula la unión y permite generar unas expectativas tanto privadas como públicas acerca de lo que se puede esperar o no de la familia; y 3) una reciprocidad o relacionalidad concreta -relazione- que da lugar a la relación-familia como realidad emergente y que es generadora de bienes o males relacionales, de entre los cuales el bien relacional primario por excelencia son los hijos que conforman la tríada familiar. Igualmente, la relación-familia es observable también en sus tres registros temporales: la familia como interacción, la familia como relación y la familia como realidad atemporal o simbólica.

Lo que caracteriza al siglo XXI, y es por lo que se teoriza acerca de la crisis o muerte de la familia, es la proliferación de nuevas y cada vez más diversas formas familiares que son el resultado de procesos morfogenéticos que no tienen por qué ser de suyo negativos, sino que en todo caso apuntan una y otra vez a esa estructura latente de la familia originaria y original (Donati, 2013) y a la posibilidad de que su naturaleza relacional esté de verdad cambiando. Por ello conviene entender por qué y en qué sentido estamos asistiendo a cambios en la familia y en qué grado están modificando o eliminando esa estructura triádica de 'plenitud relacional' propia únicamente de este sistema social.

\section{El genoma social de la familia}

Esa estructura triádica latente es lo que Donati denomina el 'genoma social' de la familia y que constituye el patrón cultural universal, estable e invariable a lo largo de la historia y en las diferentes sociedades (Donati, 2014): su relación-raíz, núcleo constitutivo de la familia. Este es luego susceptible de concretarse a nivel 
manifestativo de diversas formas, siempre contingentes, según la dualidad seracontecer o inmanencia-trascendencia: su relación-contingencia (Donati, 2014). Dicho genoma confiere a la familia identidad social al mostrar sus cualidades relacionales únicas y concretas que solo ese sistema social es capaz de generar, distinguiéndose del resto de formas de relación.

Los neofuncionalistas describen la familia actual como un 'subsistema especializado en el amor', en el que ser familia es simplemente la común problematización del mundo (Luhmann, 1985), lo cual introduce la indeterminación de las formas (Belardinelli, 2013). La sociología relacional, en cambio, identifica los cuatro elementos que constituyen ese genoma social que da forma a la relación-familia y le confiere una naturaleza única y concreta (Donati, 2008a):

- Don: propio del amor ablativo o agápico, gratuito, dirigido a un quién y no a un qué. Es principio dinamizador del genoma y de la relación entre los cuatro elementos. El don en la familia se manifiesta en la disposición de la persona siempre única e irrepetible que trasciende su propia individualidad para entregarse y servir a los demás. Ese ponerse al servicio de los otros se transfiere luego a la sociedad, generando comunidades de personas y relaciones humanas basadas en la solidaridad, la justicia y el bien común.

- Reciprocidad: es ese circuito de donaciones entre aquellos que se reconocen unidos y comprometidos por un vínculo. En este caso se trata de la reciprocidad plena entre sexos y generaciones, consecuencia directa de la donación que nutre y fortalece a la familia. Fuente de solidaridad social y de intercambios suprafuncionales, que van más allá del interés individual y la utilidad.

- Sexualidad: propia del amor esponsal, exclusiva y excluyente. No se queda únicamente en la dimensión reproductiva; sirve además de fundamento para las relaciones humanas y posiciona existencialmente a la persona en su condición sexuada para la vida en pareja, en familia y en sociedad.

- Generatividad: es la concreción de la reciprocidad entre los sexos, cuya finalidad es generar los bienes relacionales primarios que son los hijos. Mediante la generatividad, la pareja excede su individualidad, la trasciende y se convierte en comunidad generativa de amor. No se ve por tanto reducida a la procreación -generar hijos- ni al habitar, sino que es generadora de relaciones (Donati, 2014).

La combinación relacionalmente articulada de estos cuatro elementos es necesaria para dar lugar a la realidad sui géneris que conforma la familia. Efectivamente, cada una de las cuatro puede relacionarse y articularse de diversa forma, asumiendo un mayor o menor peso, pudiendo unirse entre ellas más o menos estrechamente. Pero 
la modificación, separación o desligamiento de estos cuatro elementos supondría modificar o incluso eliminar el genoma social de la familia. Y hablaríamos por tanto de modificar su principio generativo-de relaciones concretas y únicas no presentes en otros sistemas sociales de la manera como aquí se manifiestan- y acabar con su identidad, dando lugar a otras formas de convivencia o agrupamiento que, pudiendo ser metáfora o analogía de la familia, no son propiamente familia, sociológicamente hablando.

\section{El principio de la familia, relación entre origen y horizonte}

Este genoma, que representa la estructura relacional de la familia, constituye el 'horizonte cultural' y el 'principio' desde los que es posible reinterpretar y comprender la morfogénesis familiar (Donati, 2014). En primer lugar, es preciso saber que el horizonte marca el límite de aquello que se puede conocer y al mismo tiempo hace de frontera con lo que trasciende el propio conocimiento de la realidad. Partiendo de ahí, el horizonte de la familia, efectivamente, puede desplazarse cuantas veces se quiera, pero detrás siempre habrá otro. Es condición necesaria de la existencia humana, la cual es proyectiva y está siempre abierta a la realización de su latencia (Donati, 2009). Pero ese horizonte que es visto como límite o vínculo, es también recurso desde el enfoque morfogenético, en la medida en que hace posible que el hombre pueda trascender los propios límites.

Donati señala que la modernidad se ha caracterizado en este caso por el aplazamiento sistemático de los horizontes de la familia, reforzando su carácter contingente. Sin embargo, la familia tiene una peculiaridad que no posee el resto de las formas sociales, y es la de que jamás está incluida del todo en el horizonte, sino que se sitúa en el límite, tendiendo siempre a superarlo, a excederlo. Es el trascender que manifiesta la relación-familia que nunca está determinada ni contenida en su totalidad en su forma originaria, sino que es capaz de generar nuevas formas adaptativas susceptibles o no de ser institucionalizadas (Donati, 2014). El horizonte de la familia es entonces un abanico de posibilidades siempre abiertas que le permiten trascender su relacionalidad natural, su propio genoma social; de ahí la pluralización de formas de la que hoy somos testigos de forma más llamativa. Y el horizonte presente apunta siempre a otro más amplio que engloba los horizontes precedentes, considerados cada uno en su momento insuperables desde el entendimiento humano.

Todos esos horizontes siempre más amplios, son cada uno en su momento y lugar la novedad que necesita un 'principio' al que hacer referencia. Ello, porque "lo 'nuevo' no indica en absoluto lo inédito, lo imprevisto, lo totalmente distinto respecto de lo que ya se conoce, sino que apunta más bien a un modo de renovar (de 
tomar nueva) una posibilidad" (D'Agostino, 1991, p. 18). El principio es el punto de origen desde el que nacen los nuevos horizontes, es fuente del ser y motor de ese despliegue manifestativo que más que nunca muestra a la familia como sociedad natural. Y aun cuando se desplace también ese punto de referencia-como parece ser el propósito de las teorías que plantean la crisis de la familia tradicional-, la relación entre origen y horizonte sigue ahí, porque dicha relación entre origen y horizonte es el mismo principio.

Este principio lo denomina Donati 'dopomoderno', y sirve al individuo para observar la relación-familia más allá del presente y del horizonte actual en el que se enmarca. De esta manera, al introducir el concepto de genoma social de la familia es posible entender que la familia sigue y seguirá siendo una institución social actual que se puede reproducir de formas diversas, generando nuevas relaciones desde el punto de vista morfogenético. Pero todas ellas remitirán una y otra vez al mismo principio del cual son originarias.

Con todo ello se abre la vía para un conocimiento irrestricto de la familia que desde un punto de vista relacional y morfogenético siempre puede trascender su propia relacionalidad constitutiva, traspasando los límites de cualquier horizonte que de otro modo la constreñirían. De ahí la afirmación de que la familia sea un horizonte generativo, que se desplaza siempre con las personas, como matriz de nuestro mismo ser en el mundo (Donati, 2014).

\section{La mediación social de la familia}

Una vez analizado el genoma social de la familia, interesa ahora ver por qué y para qué necesita la sociedad la familia y sus bienes relacionales. Siempre desde un punto de vista sociológico, la teoría relacional se propone mostrar cómo la familia es esa "relación social de la que depende la realización de la humanidad de la persona y de la entera sociedad. Es el bien relacional primario" (Donati, 2014, p. 17).

En primer lugar, es preciso señalar que la conexión realizada de una determinada manera entre los cuatro elementos del genoma social funda el sentido de la sociabilidad de quienes conforman la familia. Es decir, el modo como se es y se hace familia deja una impronta tanto en la construcción de la propia identidad como en la posterior socialización de las personas.

Por socialización se entiende la adaptación a la sociedad en la que se vive, lo que se aprende en primer lugar en la familia, conformación que hay que reforzar si la sociedad a la que es necesaria hacerse está, como 
ocurre hoy, en constante cambio por factores nocivos o por circunstancias de progreso, entendido en el mejor de los sentidos. (Altarejos, Bernal y Rodríguez, 2009, p. 176)

En este sentido, la familia ejerce una triple mediación desde el punto de vista relacional (Donati, 2003):

1. Entre naturaleza y cultura: la familia media para la transformación de la naturaleza interna del individuo en obrar cultural, a través de un proceso en el que los elementos biológicos y psíquicos constitutivamente humanos son civilizados para el despliegue de su sociabilidad. Así, la familia hace accesibles al individuo los elementos simbólicos, culturales, normativos, adaptativos y organizativos que debe tener en cuenta en sus relaciones sociales.

La sociabilidad no es una meta o resultado que se logre o encuentre, sino una búsqueda incesante de uno mismo, no en la introspección isolativa del psiquismo, sino en la apertura radical a los otros mediante la aceptación-donación, que se actualiza en la praxis cotidiana de la convivencia familiar. (Altarejos, Bernal y Rodríguez, 2009, p. 181)

La familia es el lugar donde el individuo descubre su propia identidad personal, familiar y social, proceso que no tiene lugar ni desde la autonomía (semántica moderna) ni desde la confrontación o la negación (semántica posmoderna), sino desde la relación que origina el encuentro con la alteridad (semántica relacional). "La persona es ese status que atribuimos el uno al otro en la relación" (Allodi, 2007, p. X). Dependiendo del 'sentido familiar' que se viva en la familia y del nivel de institucionalización de la misma, el individuo podrá construir su propia identidad a través de relaciones que le ofrecerán mayor o menor estabilidad, previsibilidad y capacidad de maduración (Donati, 2013), lo cual condicionará el tipo de aportaciones que este pueda hacer en la esfera privada y pública y las relaciones que vaya a crear con los demás y con su entorno.

2. Entre individuo y sociedad: sobre todo en la actualidad, la sociedad globalizada obliga a la familia a reforzar y aumentar su función de mediación. Media respecto de sus posibilidades y modos de participación en la esfera pública, dependiendo de su nivel de institucionalización y del reconocimiento que tenga como sujeto social. Su rol de mediadora entre lo interno y lo externo crece paralelamente al aumento del número y diversidad de agencias de mediación externa que también interceden entre el individuo y la sociedad, precisamente para tener conocimiento y control de todo aquello a lo que se puede ver expuesta la persona. 
En este sentido, la familia acentúa sus funciones de mediación, y es ahí donde la educación debería intervenir como función de reflexión, organización y respuesta a las necesidades de la persona humana, que se ve imbricada en un juego de ligámenes e interacciones demasiado complejas para ella. (Donati, 2014, p. 125)

3. Entre esfera pública y privada: tarea que cada vez se hace más compleja con el entrelazamiento de los mundos de vida y el sistema social en la propia familia. Belardinelli (1996) llama la atención sobre el fenómeno de privatización y 'publicación' - en el sentido de hacerse cada vez más asunto público- de la familia, ya sea porque el ordenamiento jurídico ya no regula prácticas o fenómenos que antes quedaban circunscritos al ámbito familiar (adulterio, separación, etc.), o porque comienzan a regularse ciertas prácticas que ahora se consideran de interés público por su repercusión en las dinámicas sociales (adopción, custodia, separación de bienes, etc.). La familia está llamada a rediseñar las relaciones internas y externas con la sociedad, también con el objetivo de eliminar la brecha entre esfera privada y pública que todavía ciertos autores insisten en señalar (Caporrella, 2008).

Esta revisión de las funciones mediadoras de la familia permite a Donati desmontar la creencia generalizada a lo largo del siglo XX de que la familia perdía funciones sociales (Donati, 2014), así como la idea extendida por ciertas ideologías en las décadas de 1960 y 1970 de que la familia comenzaba a ser obstáculo para la libertad, la igualdad, la autorrealización, la justicia y la emancipación (Belardinelli, 1996). Belardinelli (1996) señala que, a pesar de que algunas de las funciones primarias de la familia - crianza, cuidado, socialización, educación, asistencia a personas dependientes, etc.- han disminuido por delegación en instituciones y agencias externas, al mismo tiempo asume otras nuevas como resultado de los cambios socioculturales y económicos que la sociedad experimenta. Ello, si bien desarrolla su papel de mediación social a través de nuevas modalidades que difieren respecto de épocas anteriores. Pero la institución familiar sigue siendo esa realidad relacional necesaria y que de hecho existe porque así lo exigen los individuos y la misma sociedad para hacer accesible y familiar ese mundo que no es directamente familiar - no es inmediatamente humano. "Esta exigencia, tan honda como inconsciente, implica un proceso aparentemente paradójico de distanciamiento e inclusión entre familia y sociedad" (Donati, 2014, p. 124).

La familia ejerce por tanto una función mediadora de doble direccionalidad: hacia el interior de su propia estructura a través del intercambio de bienes relacionales y educación de las generaciones, y hacia el exterior transfiriendo a la sociedad 
esos bienes que contribuyen a la creación de relaciones humanas, sólidas y estables, generadoras de capital social a través de la socialización de sus miembros. La familia se convierte así en la clave para hacer lo social, familiar; para distinguir lo humano de lo no-humano y humanizar las relaciones sociales, y contribuir con ello a la generación de una cultura orientada al cuidado, como de hecho hace (Donati, 2013).

Como consecuencia, la educación familiar se hace crucial y al mismo tiempo compleja, también por las interconexiones que se van creando entre familia y sistemas sociales (centros educativos, escuelas de tiempo libre, centros cívicos, academias, instituciones sociales, etc.), más allá de la autonomía sistémica (Belardinelli, 1996). El condicionamiento recíproco entre familia y sociedad es manifestación de esa mediación social y cultural con carácter bidireccional que puede ser de corto-medio alcance-comunitaria- o extensible a la sociedad en general -societaria- (Donati, 2003).

Lo que la sociedad debe plantearse es cómo se puede ayudar a la familia a ser cada vez más ella misma, a cumplir con las funciones de mediación de las que depende la socialización de sus miembros y, en definitiva, a la creación de una sociedad más humana, más familiar. Esto pasa por el reconocimiento de su subjetividad -libertad y responsabilidad, derechos y obligaciones- en tanto agente de socialización y educación, pero además en cuanto agente de cambio y transformación social, lo cual reclama la institucionalización de la familia como sociedad natural y sistema social (Donati, 2013).

\section{Conclusiones}

La historia demuestra que la familia normo-constituida mantiene una continuidad más o menos estable a lo largo de las épocas y en las distintas sociedades, si bien se manifiesta en cada caso de manera diversa. Su existencia pone en evidencia una estructura latente que, más allá de roles y funciones, apunta a relaciones. Esa relacionalidad concreta que le es propia justifica por sí sola la existencia y la funcionalidad de la familia como institución social única. Pero las teorías que insisten en la crisis y muerte de la familia no parecen ver más allá del horizonte cultural que en este caso muestra los profundos cambios a los que se enfrenta este sistema social.

La sociología relacional propone un cambio de enfoque para la interpretación de la pluralización de las formas familiares desde un punto de vista relacional y morfogenético. Esto pasa por entender, en primer lugar, qué es y por qué existe la familia en su sentido sociológico, qué funciones desempeña y para qué es necesa- 
ria en la sociedad. Para responder a estos interrogantes ha sido necesario analizar su estructura relacional y su capacidad generativa interna y externa. Ello, con el objetivo de señalar que la familia efectivamente cambia, genera y se regenera, se adapta, pero permanece invariable el principio que da origen a nuevos horizontes socioculturales. Ese principio basado en el amor gratuito, entendido como don, es fuente de relaciones e intercambios que son más que nunca necesarios en una sociedad individualizada basada en el interés, la utilidad y el resultado inmediato.

La reinstitucionalización de la familia y el reconocimiento de su subjetividad social como agente de socialización, de educación y también de cambio y transformación social, se hacen imprescindibles. La familia se encuentra en la actualidad en tensión entre su privatización y el ser cada vez más un asunto de interés público, pero desplazada por los sistemas político y económico que afianzan la separación entre la esfera pública y privada. Por eso la sociología relacional de Donati pretende mostrar que la relacionalidad familiar exige que se le reconozcan derechos y obligaciones que vayan más allá de la perspectiva individual: la mujer, el niño, el enfermo, el discapacitado, el mayor, etc. (Donati, 2008b).

Ser institución social significa tener un 'lugar' en donde estar previsto y esperado (Donati, 2014). Teniendo en cuenta esa relación de interdependencia entre familia y sociedad, todo parece indicar que la desinstitucionalización de la familia y su eliminación como sociedad natural tendría a la larga serias implicaciones en la forma como se genera y regenera la sociedad y sus intercambios. En palabras de Becerril, "la amenaza o la crisis de la familia no está en la pérdida de funciones o en su diversidad morfológica, la familia puede desintegrarse si llega a fragmentarse, alcanzando la total individualización de las personas que la componen" (2006, p. 82). Ello, con la consiguiente generalización de males relacionales que hoy se manifiestan en patologías sociales con efectos devastadores en las personas, las familias y la entera sociedad: no ser amados o aceptados, ser amados por un amor equivocado, sufrir violencia de todo tipo (Larraín, 2004), ser explotados y considerados como cosas, "son tantas degeneraciones que pertenecen lamentablemente a la ordinaria fenomenología de la vida familiar" (Belardinelli, 2013, p. 87).

Pero la evidencia empírica dice que el sistema político es cada vez más insensible, incapaz e inadecuado para llevar a cabo la tarea de promover la familia [...]. El futuro estará marcado por la necesidad de un vuelco cultural en las políticas educativas y sociales, para salir del asistencialismo como modelo dominante [...]. Debemos pensar en un nuevo horizonte, en acciones de la sociedad civil que tengan como protagonistas a las familias [...]. (Donati, 2014, p. 126) 
El sistema social tiene la responsabilidad de hacer visibles y reconocer los bienes relacionales que solo la familia genera y transfiere a la sociedad. Bienes que van más allá de las habilidades cognitivas y competencias emocionales y cívicas que tan en boga están actualmente; implican el dar y recibir confianza, saber cooperar con los demás por el hecho de ayudar, aprender y practicar las reglas del don y la reciprocidad no instrumental, todas ellas virtudes en las que se basa la convivencia civil (Donati, 2014). Y que a su vez repercuten en la esfera pública: sentido de pertenencia a las organizaciones, compromiso con las obligaciones, lealtad a las empresas, confianza en las personas, relaciones de compromiso de todo tipo, responsabilidad social, solidaridad, transformación y mejora social, etc.

La familia está llamada en este sentido a asumir el papel de agente educativo que puede y debe jugar un papel crucial en la educación y socialización de las nuevas generaciones, y en la creación y promoción de una cultura orientada al cuidado de las personas y el entorno. 


\section{Referencias bibliográficas}

Allodi, L. (2007). La trascendenza, "luogo" dell'umano. En R. Spaemann, Persone. Sulla distinzione tra "qualcosa” e "qualcuno" (pp. V-XV). Bari: Gius, Laterza y Figli Spa.

Altarejos, F., Bernal, A., Rodríguez, A. (2009). La familia, escuela de sociabilidad. Educación y Educadores 8, 173-185.

Archer, M. S. (2007). La morfogenesi della società. Milano: Franco Angeli.

Ayuso Sánchez, L. (2010). Juventud y familia en los comienzos del siglo XXI. En P. González Blasco, J. González-Anleo (dirs.), Jóvenes españoles 2010K (pp. 117-174). Madrid: SM.

Bachofen, J. J. (1992). El matriarcado: una investigación sobre la ginecocracia en el mundo antiguo según su naturaleza religiosa y jurídica. Madrid: Akal.

Bauman, Z. (2001). La sociedad individualizada. Madrid: Cátedra.

Becerril, D. (2006). Las relaciones familiares mediadas: nuevos medios y viejas pautas en el cambio social. En Centro de Estudios Andaluces, El cambio social en España. Visiones y retos del futuro (pp. 71-85). Sevilla: Consejería de la Junta Presidencial, Junta de Andalucía.

Beck, U. (1996). Il normale caos del amore. Torino: Boringhieri.

Beck, U., Beck-Gernsheim, E. (2002). Individualization. Institutionalized Individualism and its Social and Political Consequences. London: Sage.

Belardinelli, S. (1996). Il gioco delle parti. Identità e funzioni della famiglia in una società complessa. Roma: Veritas Editrice.

(2013). La familia como recurso de una sociedad abierta y plural. Estudios Sobre Educación 25, 85-94.

Berger, B., Berger, P. L. (1983). In difesa della famiglia Borghese. Bologna: Il Mulino.

Bernal, A., Rodríguez, A., Altarejos, F., Naval, C. (2005). La familia como ámbito educativo. Pamplona: Rialp.

Boas, F. (1964). Cuestiones fundamentales de antropología cultural. Buenos Aires: Solar/Hachette.

Cabezas, A. M. (2008). La separación en la familia. Su observación como sistema. Persona y Sociedad 22 (1), 41-58.

Caporrella, V. (2008). La famiglia. Un'istituzione che cambia. Bologna: Archettipolibri.

Crespo Garrido, M., Moretón Sanz, M. F., eds. (2010). Discriminación por razón de edady de sexo. Retos pendientes del Estado Social. Madrid: COLEX.

D’Agostino, F. (1991). Elementos para una filosofía de la familia. Madrid: Rialp.

De Rougemont, D. (2006). El amor y Occidente. Barcelona: Kairós.

Del Campo, S. (2006). Cambio social y familia. En Centro de Estudios Andaluces, El cambio social en España. Visiones y retos del futuro (pp. 39-57). Sevilla: Consejería de la Junta Presidencial, Junta de Andalucía.

Díez Nicolás, J. (1983). La familia en Europa y el cambio social. Reis 21, 11-31. 
Donati, P. (1983). Introduzione alla sociologia relazionale. Milano: Franco Angeli. (1986). La famiglia nella società relazionale: nuove reti e nuove regole. Milano: Franco Angeli.

(1987). Famiglia e politiche sociali: la morfogenesi familiare in prospettiva sociologica. Milano: Franco Angeli.

(1989). La famiglia come relazione sociale. Milano: Franco Angeli. (2003). Manual de sociología de la familia. Barañáin: EUNSA. (2008a). Perché “la” famiglia? Le risposte della sociologia relazionale. Siena: Edizioni Cantagalli.

(2008b). Oltre il multiculturalismo. La ragione relazionale per un mondo comune. Roma-Bari: Editori Laterza.

(2009). La società dell'umano. Genova-Milano: Casa Editrize Marietti. (2010). Reflexivity after Modernity. From the Viewpoint of Relational Sociology. En M. S. Archer (ed.), Conversations About Reflexivity (pp. 144-164). New York: Routledge. (2013). La familia como raiz de la sociedad. Madrid: Biblioteca de Autores Cristianos. (2014). La familia. El genoma de la sociedad. Madrid: Rialp.

Donati, P., Colozzi, I. (1997). Giovanni e generazioni. Quando si cresce in una società eticamente neutra. Bologna: Il Mulino.

Donati, P., Solci, R. (2011). I beni relazionali. Che cosa sono e quali effetti producono. Torino: Bollati Boringhieri.

Fernández Muńoz, J. N. (2000). Los mayores y la familia. Transformaciones sociales en España en las dos últimas décadas. En S. Adroher Biosca (coord.), Mayores y familia (pp. 19-36). Madrid: Instituto Universitario de la Familia, Universidad Pontificia Comillas de Madrid e IMSERSO.

García Garrido, J. L., coord. (2000). La sociedad educadora. Madrid: Fundación Independiente.

Giddens, A. (1992). The Transformation of Intimacy: Sexuality, Love and Eroticism in Modern Societies. Cambridge: Polity Press.

Gil, W. E., Arias, M. Y. (2013). La pluralidad familiar, a la luz de la sociología relacional. Anfora 20 (35), 173-195.

Héritier, F. (1984). L'esercizio della parentela. Roma: Laterza.

Larraín, S. (2004). Violencia en la familia. Persona y Sociedad 18 (1), 11-30.

Luhmann, N. (1985). El amor como pasión. Barcelona: Ediciones 62.

Martín López, E. (1993). Textos de sociología de la familia. Una relectura de los clásicos (Linton, Tönnies, Weber y Simmel). Madrid: Ediciones Rialp.

Naval, C., coord. (2003). Respuestas del grupo de investigación en educación para la ciudadanía (GIEC) de la Universidad de Navarra. Revista de Educación, Número Extraordinario, 425-442. 
Putnam, R. D. (2003). El declive del capital social: un estudio internacional sobre las sociedades y el sentido comunitario. Barcelona: Galaxia Gutenberg.

Roussel, L. (1980). Mariages et divorces. Contribution à un analyse systematique des modèles matrimoniaux. Population (Nov.-Dic.), 1025-1040.

Terenzi, P. (2006). Sociologia relazionale e realismo critico. En Donati, P., Terenzi, P., Invito alla sociologia relazionale: teoria e applicazioni (pp. 33-49). Milano: Franco Anteli. 
